\title{
Optimization of Aflatoxin B1 Aptasensing
}

\author{
Marzieh Jafari, ${ }^{1}$ Mohsen Rezaei, ${ }^{1,2}$ Heibatullah Kalantari, ${ }^{1}$ \\ Maryam Tabarzad, ${ }^{3}$ and Bahram Daraei ${ }^{2}$ \\ ${ }^{1}$ Department of Pharmacology and Toxicology, School of Pharmacy, Ahvaz Jundishapur University of Medical Sciences, Ahvaz, Iran \\ ${ }^{2}$ Department of Toxicology, Faculty of Medical Sciences, Tarbiat Modares University, Tehran, Iran \\ ${ }^{3}$ Protein Technology Research Center, Shahid Beheshti University of Medical Sciences, Tehran, Iran
}

Correspondence should be addressed to Mohsen Rezaei; rezaei.mohsen@gmail.com and Bahram Daraei; bdaraei@modares.ac.ir

Received 2 January 2017; Accepted 19 April 2017; Published 14 May 2017

Academic Editor: Orish Ebere Orisakwe

Copyright (C) 2017 Marzieh Jafari et al. This is an open access article distributed under the Creative Commons Attribution License, which permits unrestricted use, distribution, and reproduction in any medium, provided the original work is properly cited.

\begin{abstract}
Combination of aptamers with DNAzymes attracted intense attention for development of DNA-based biosensors for detection of mycotoxins. In the present study a combination of aflatoxin B1 specific aptamer and HRP- (horseradish peroxidase-) mimicking DNAzyme was optimized for detecting aflatoxin B1. Detecting approach is based on the binding affinity of aflatoxin B1 to its specific aptamer and conversion of substrate to a detectable colorimetric signal by a linked DNAzyme. Compared to conventional methods for aflatoxin B1 detection, DNA-based assay has the advantages of low cost, long-term stability, and rapid, simple, and user-friendly steps.
\end{abstract}

\section{Introduction}

Mycotoxins are the well-known secondary metabolites of fungi with established adverse health effects in human and animals [1]. Aflatoxins are a group of mycotoxins produced by a variety of Aspergillus species including A. flavus and $A$. parasiticus [2]. Human contacts to these toxic metabolites are inevitable during preharvest, storage, or processing of agricultural products [3]. AFB1, the most toxic form of aflatoxins, has been linked to various health problems and has been placed in group 1 of human carcinogens by the International Agency for Research on Cancer (IARC) [4]. Food safety strategies and imminent contamination of crops with aflatoxins have increased the importance of AFB1 detection worldwide.

Various analytical methods have been developed for determination of AFB1 levels in food stuffs [5]. Despite their sensitivity and accuracy, chromatography based methods met some limitations that can be time consuming and expensive from sample preparation to the detection steps. Disadvantages of protein based assays are stability of antibodies under strict physical and chemical conditions and their long-term production processes. Consequently it is required to develop a rapid, simple, and low cost technique for AFB1 detection in food samples.

Numerous DNA sequences with enzymatic functions besides carrying of genetic information have been identified $[6,7]$. Single-stranded DNAs because of their folding properties have been enrolled in many analytical procedures for developing of several DNA-based biosensors [8, 9].

The term aptamer refers to a single-stranded DNA which folds in to three-dimensional structure and interacts with its specific target [10]. This specific and short single-stranded oligonucleotide is chosen from a random sequence library and can bind to a wide variety of small molecule targets including proteins, nucleic acids, and cell receptors [11, 12]. DNAzyme, also known as deoxyribozyme, refers to a guanine-rich nucleic acid sequence with catalytic capabilities similar to common enzymes [13]. Several single-stranded DNA sequences with enzymatic activity in various colorimetric reactions have been identified [14].

DNAzymes and aptamers have remarkable advantages, including of low cost preparation and high efficiency $[15,16]$. They are stable in different chemical or physical conditions. In contrast to proteins, they return to their original conformations when $\mathrm{pH}$ and temperature return to the initial 
TABLE 1: Sequences of oligonucleotides used in this study. ssDNA: single stranded DNA; B: blocker complementary sequence.

\begin{tabular}{ll}
\hline ssDNA & Sequences $\left(5^{\prime}-3^{\prime}\right)$ \\
\hline $\begin{array}{l}\text { DNAzyme- } \\
\text { aptamer }\end{array}$ & TGGGTAGGGCGGGTTGGGAAAGTTGGGCACGTGTTGTCTCTCTGTGTCTCGTGCCCTTCGCTAGGCCCAC \\
$\begin{array}{l}\text { Aptamer- } \\
\text { DNAzyme }\end{array}$ & GTTGGGCACGTGTTGTCTCTCTGTGTCTCGTGCCCTTCGCTAGGCCCACAAATGGGTAGGGCGGGTTGGG \\
B1 & CACGTGCCCAACAAATCCCAACCC \\
B2 & CTGACAGAGAGAAACCACGTGCCCAACAAATCCCAACCC \\
B3 & GAGAGACAACACGTGCCCAACAAATCCCAACCCGCC \\
\hline
\end{tabular}

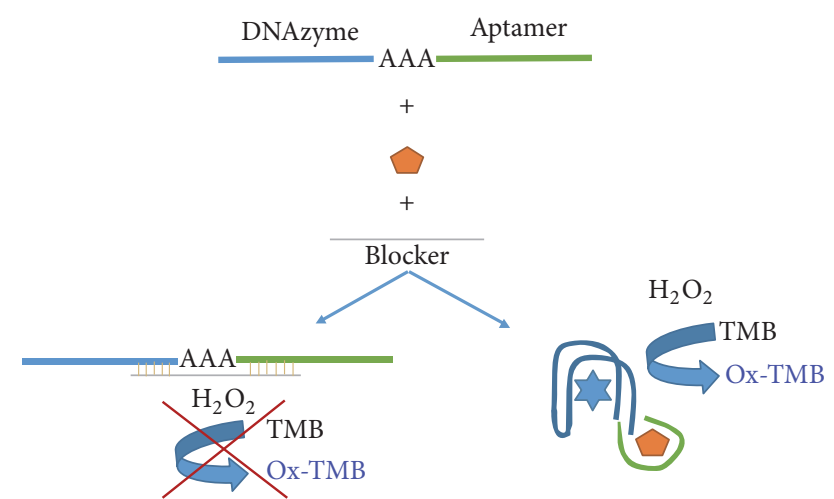

AFB1

Hemin

Scheme 1: Schematic representation of AFB1 detection by conjugated DNAzyme-aptamer.

condition $[17,18]$ and their combination has been recruited to analyze a wide range of molecules [19-22]. Based on these properties, many DNA-based biosensors have been introduced for detection of mycotoxins $[23,24]$.

In the present study a combination of AFB1 specific aptamer [25] and HRP- (horseradish peroxidase-) mimicking DNAzyme [13] was optimized for detecting of AFB1 (Scheme 1). Binding of AFB1 to its specific aptamer recognition sequence prevents the blocker from being annealed to the aptamer and the reaction proceeds to yield a blue color product in a concentration dependent manner.

\section{Materials and Methods}

2.1. Reagents. Aflatoxin $\mathrm{B} 1$ (AFB1), Tris $\mathrm{HCl}$, sodium chloride $(\mathrm{NaCl})$, magnesium chloride $(\mathrm{MgCl} 2)$, 3,3,5,5-tetramethylbenzidine (TMB) are purchased from Sigma (USA). Hemin was purchased from Serva (USA). All the chemical reagents were of highest grade and used without further purification. All solutions were prepared with diethyl pyrocarbonate (DEPC) treated deionized water.

Oligonucleotides contained a sequence of $49 \mathrm{bp}$ aflatoxin B1 aptamer [25] and 18 bp DNAzyme [13] in two different sequential orders $\left(5^{\prime}\right.$-aptamer-DNAzyme- $3^{\prime}$ or $5^{\prime}$-DNAzymeaptamer- $\left.3^{\prime}\right)$ and blockers complementary sequences were purchased from Biolegio (Netherlands). These sequences are

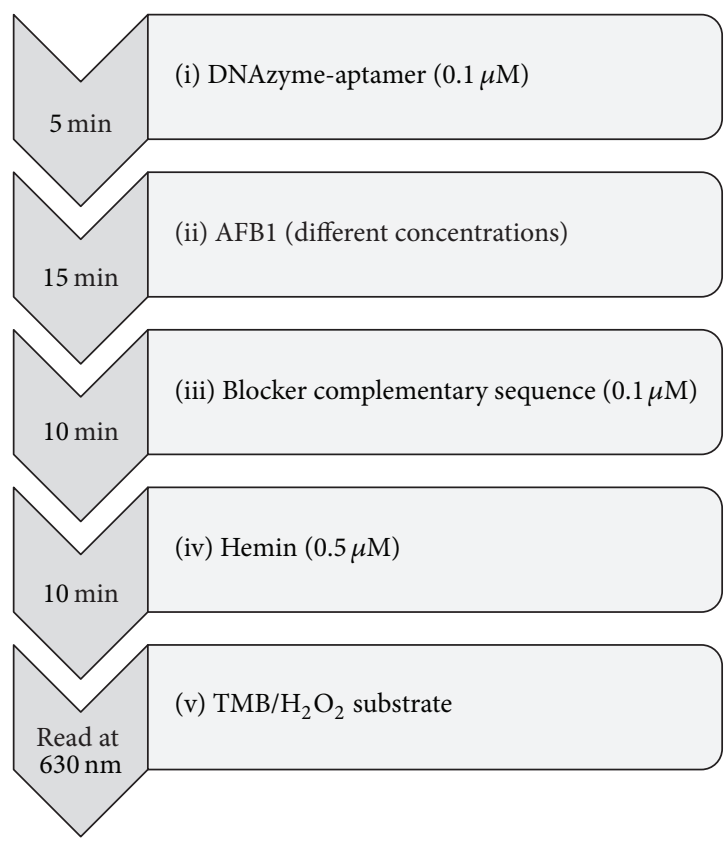

SCHEME 2: Schematic representation for optimized assay procedure.

shown in Table 1. Mfold software was used for prediction of the secondary structure of used single-stranded nucleic acids [26].

2.2. Assay Procedure. DNA stock solutions $(100 \mu \mathrm{M})$ were prepared in DEPC deionized water and stored in small aliquots. DNA working solutions were prepared in incubation buffer (10 mM Tris- $\mathrm{HCl}, \mathrm{pH} 8,120 \mathrm{mM} \mathrm{NaCl}, 2.5 \mathrm{mM}$ $\mathrm{MgCl} 2$, and $5 \mathrm{mM} \mathrm{KCl}$ ) [22]. Before starting the experiments oligonucleotides were denatured at $95^{\circ} \mathrm{C}$ for $5 \mathrm{~min}$. Then, $70 \mu \mathrm{L}$ of AFB1 aptamer (final concentration of $0.1 \mu \mathrm{M}$ ) incubated with different concentrations of $10 \mu \mathrm{L}$ of AFB1 for $15 \mathrm{~min}$. $10 \mu \mathrm{L}$ of $1 \mu \mathrm{M}$ blocker complementary sequence was then added in to 96-well microplate and incubated for $10 \mathrm{~min}$ at room temperature. $10 \mu \mathrm{L}$ of $0.5 \mu \mathrm{M}$ hemin was also added to wells followed by $10 \mathrm{~min}$ incubation. TMB substrate containing $\mathrm{H}_{2} \mathrm{O}_{2}$ was prepared immediately before use and $100 \mu \mathrm{L}$ of substrate solution was added to the mixture. For kinetic assay, absorbance of TMB color product was measured at wavelength of $630 \mathrm{~nm}$ for $5 \mathrm{~min}$ (Scheme 2). All 


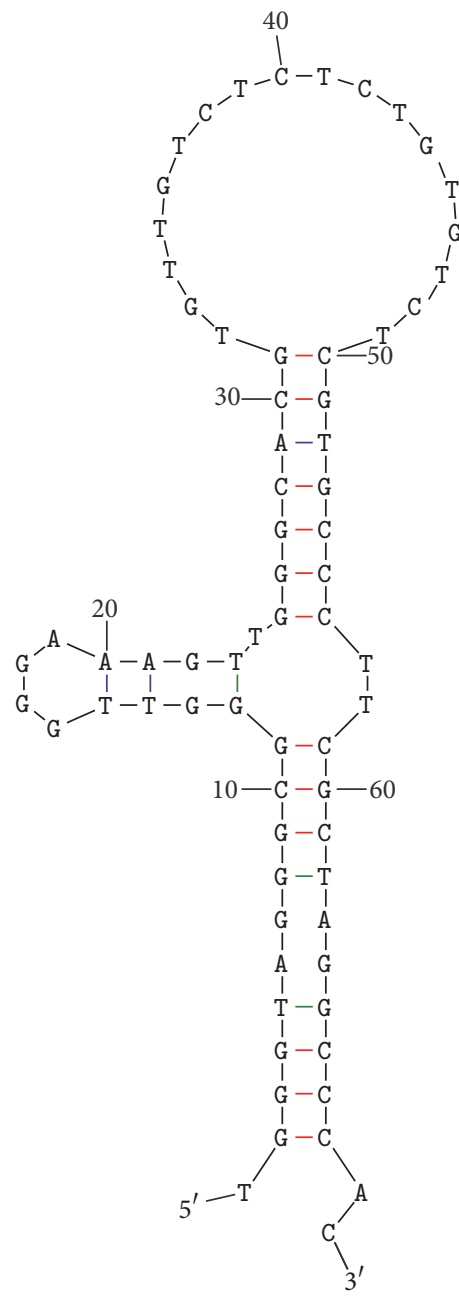

$d G=-13.14$ DNAzyme-aptamer

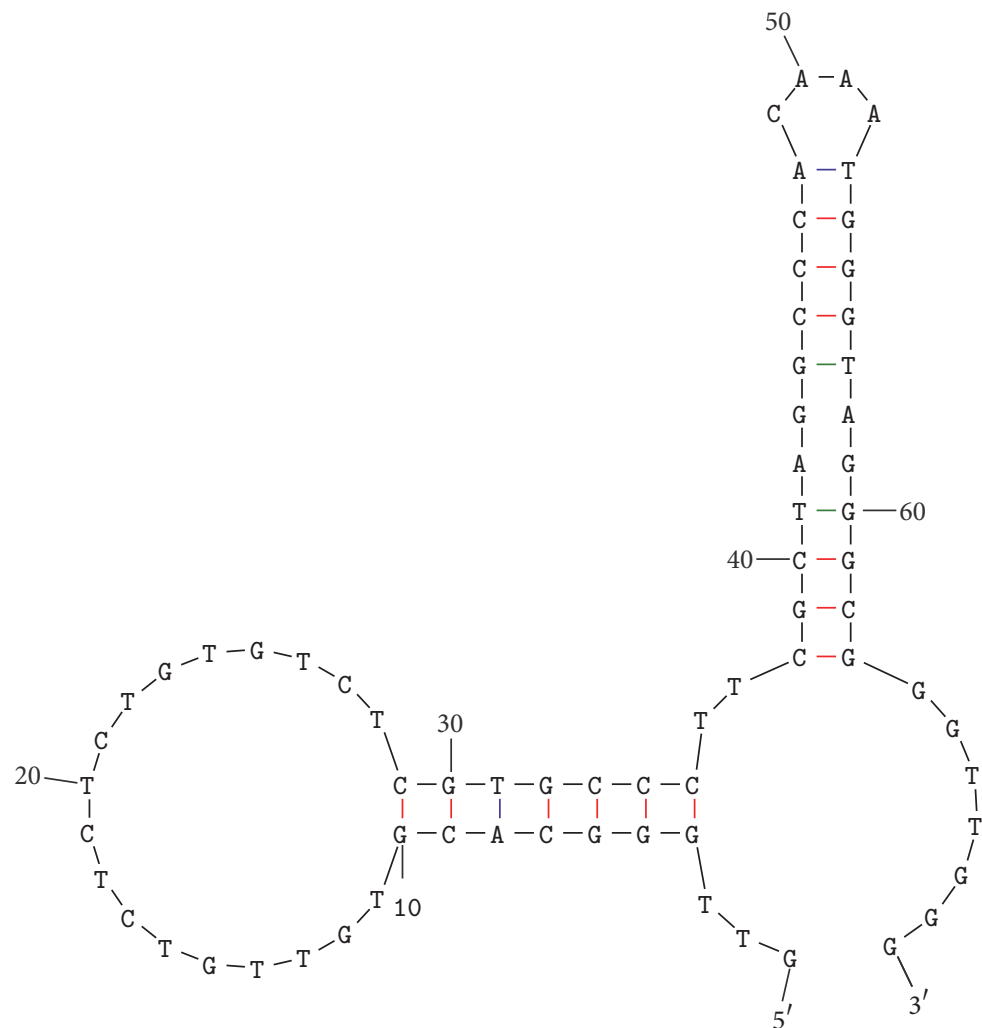

$d G=-16.95$ aptamer-DNAzyme

Figure 1: Predicted secondary structure of DNAzyme-aptamer and aptamer-DNAzyme using Mfold tool.

absorbances were measured by a BioTek (ELx800) microtiter plate reader (BioTek, USA).

\section{Results and Discussion}

DNAzyme sequence can attach to either $5^{\prime}$ or $3^{\prime}$ ends of aptamer sequence yield DNAzyme-aptamer or aptamerDNAzyme (Table 1 and Figure 1). In our previous study, we investigated the relationship between the orientation of the DNAzyme and aptamer conjugation and their final peroxidase activities [27]. As seen in Table 2, DNAzymeaptamer displayed a higher enzymatic activity than $3^{\prime}$ oriented conjugation. Aptamer-DNAzyme revealed its priority for further evaluations in biosensor design.

For achieving of best results, several parameters were optimized including blocker complementary sequences (B1, B2, and B3) selection, incubation times, reagent concentrations, and their order of addition. Optimal annealing to DNAzyme-aptamer and inhibiting of its enzymatic activity
TABLE 2: Kinetic parameters for DNAzyme-aptamer and aptamerDNAzyme catalytic activity.

\begin{tabular}{lcc}
\hline & DNAzyme-aptamer & Aptamer-DNAzyme \\
\hline$V_{\max }(\mathrm{mM} / \mathrm{s})$ & 0.06 & 0.02 \\
$K_{m}(\mathrm{mM})$ & 0.6 & 0.4 \\
\hline
\end{tabular}

were influenced mainly through the blocker complementary sequence. B1, B2, and B3 were designed with different numbers and sequences of nucleotides. As shown in Figure 2 the blockade of peroxidase activity has considerably been attained by $\mathrm{B} 3$ sequence compared to $\mathrm{B} 1$ and $\mathrm{B} 2$.

At the next step, different molar ratios of DNAzymeaptamer and B3 blocker from 1:1 to 1:5 were tested. With a constant amount of DNAzyme-aptamer $(0.1 \mu \mathrm{M})$, increasing the blocker concentration from 0.1 to $0.5 \mu \mathrm{M}$ resulted in a concentration dependent decline of DNAzyme activity (Figure 3). Given that AFB1 and complementary sequence of 


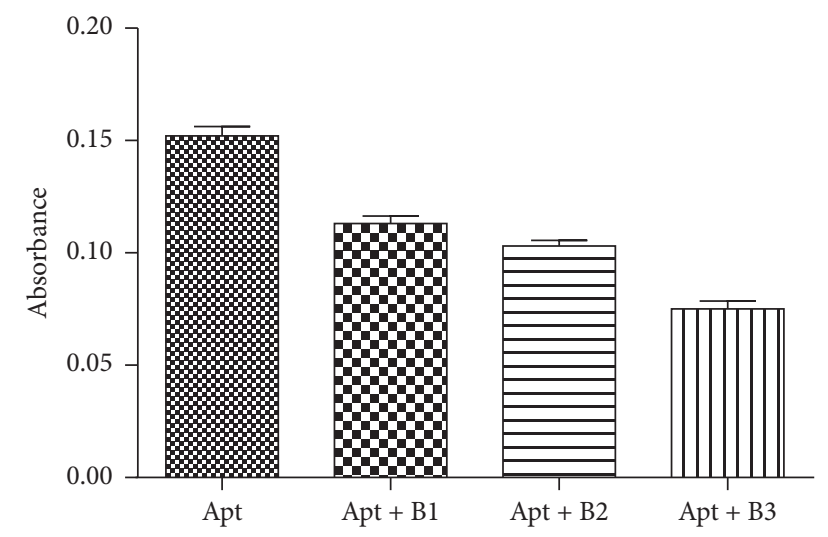

Figure 2: Blockade of peroxidase activity of DNAzyme-aptamer by B1, B2, and B3 sequences. Apt: DNAzyme-aptamer; B: blocker complementary sequence.

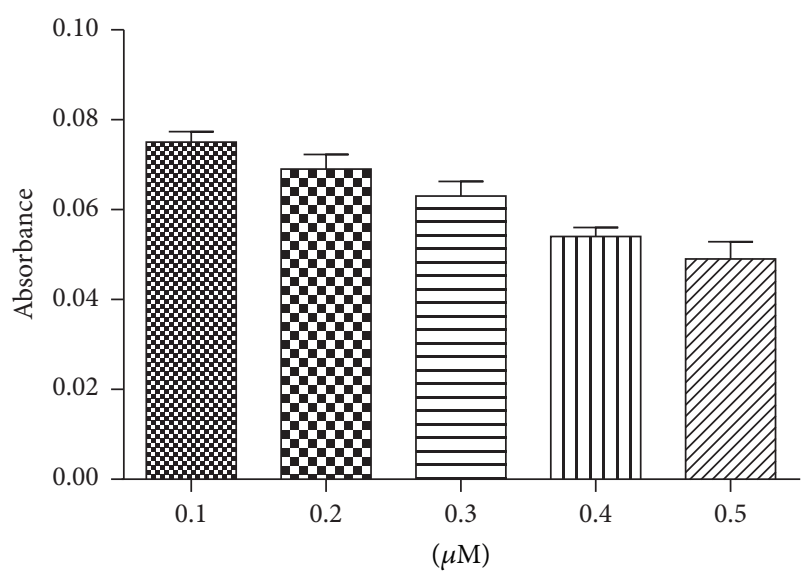

FIGURE 3: Blockade of peroxidase activity of DNAzyme-aptamer by increasing concentration of $\mathrm{B} 3$.

blocker compete with each other for binding to DNAzymeaptamer, blocker at high concentration decreases the sensitivity of assay. So a conservative lower molar ratio of $1: 1$ was selected for further evaluations.

Orders of addition of AFB1, blocker complementary sequence, and hemin were evaluated in different settings. Results showed that adding blocker prior to AFB1 resulted in unaffected remaining of blocker at its binding site. Therefore AFB1 was scheduled to incubate with aptamer before the blocker addition. Also we observed that enzymatic activity will be temporally influenced when hemin is added to the reaction containing blocker. As hemin prevented the hybridization of blocker complementary sequence and DNAzyme-aptamer, it was added subsequently to the blocker sequences addition.

The best incubation times for AFB1, blocker complementary sequence, and hemin were achieved via incubation of all reagents separately at $10 \mathrm{~min}$ intervals up to 60 minutes. The shortest and most efficient incubation times were 15, 10, and $10 \mathrm{~min}$ for AFB1, blocker, and hemin, respectively.

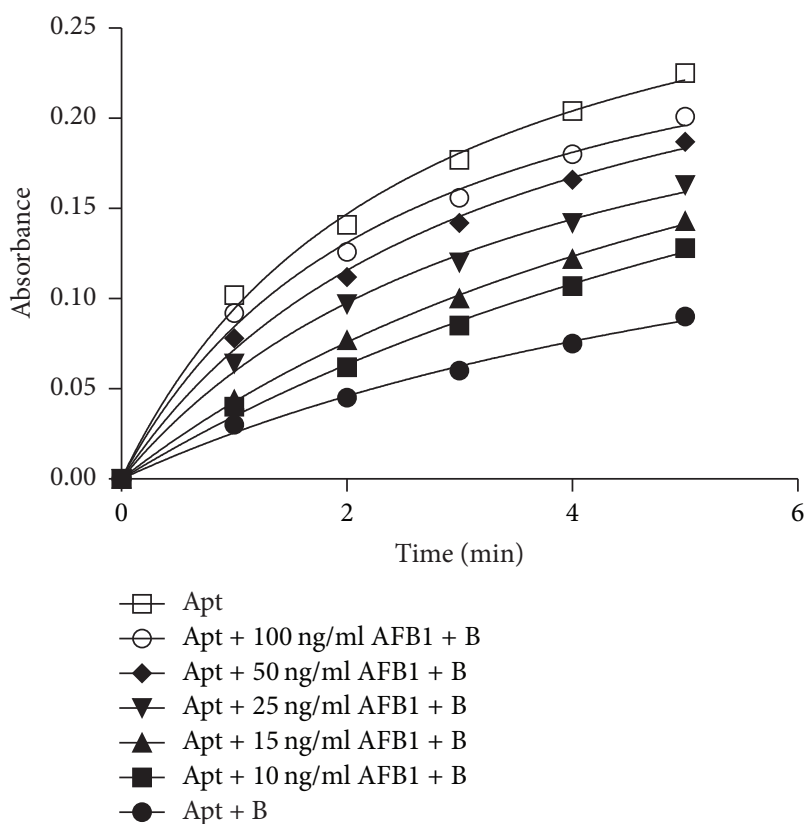

FIGURE 4: Peroxidase activity of DNAzyme-aptamer in the presence of B3 and different concentrations of AFB1. Apt: DNAzyme-aptamer, B: blocker complementary sequence, and AFB1: aflatoxin B1.

In the present work, the sensing strategy is based on the binding affinities of AFB1 to its specific aptamer that produce a detectable colorimetric signal by DNAzyme (Scheme 1). In the absence of AFB1, annealing of blocker sequence (complementary sequence to a part of DNAzyme-aptamer) to DNAzyme-aptamer decreases the enzymatic activity. In the presence of AFB1, the aptamer binds to AFB1 and forms a hairpin structure. Consequently, blocker complementary sequence was prevented from being bound to DNAzymeaptamer and following addition of hemin, DNAzyme displays a colorimetric signal that is directly associated with AFB1 concentrations (Figure 4).

Under optimal conditions the limit of detection of $10 \mathrm{ng} / \mathrm{mL}$ was achieved. AFB1 aptamer has been used as a recognition probe in several detection systems based on PCR, electrochemical, chemiluminescent, colorimetric, and fluorescent platforms. In Guo et al's study, AFB1 aptamer with $3^{\prime}$-terminal biotin groups has been immobilized on the surface of PCR tubes for developing of an aptasensor (LOD: $25 \mathrm{fg} / \mathrm{mL}$ ) based on RT-qPCR [28]. An aptamerbased dipstick assay (LOD: $0.1 \mathrm{ng} / \mathrm{mL}$ ) using biotin-modified aptamer has also been reported by Shim et al. [29]. In another work by Shim et al. based on chemiluminescence competitive assay, AFB1-OVA conjugate was coated on the wells (LOD: $0.11 \mathrm{ng} / \mathrm{mL}$ ) [30]. Castillo et al. have developed an aptamerbased biosensor (LOD: $0.40 \mathrm{nM}$ ) using immobilization of amino-modified aptamers and electrochemistry [25].

One of the important goals of this work was designing a simple and cost-effective method for $\mathrm{AB} 1$ detection, without intricate steps and equipment. In the mentioned publications, aptamer has been modified with functional groups or immobilized on surfaces. In our experiment, $\mathrm{AB1}$ aptamer has been 
employed without any immobilization and modification. All steps of procedure were done as a simple solution. Since aptamer interaction with its target depends on folding into unique structures [31], intact aptamers were excepted to have more appropriate folding.

AFB1 aptamer and DNAzyme also have also been used by Seok et al. with some alterations [32]. In their study, two split DNAzyme halves anneal with aptamer that form G-quadruplex. The AFB1 aptamer complex prevented the annealing of split DNAzyme and aptamer, therefore weak color intensity will be observed upon addition of ABTS substrate. The accuracy of this method is depending on the correct annealing of two split DNAzymes with aptamer. The amount of this annealing may be variable in each performance and cause false negative results. In our work, to have the stable signal, DNAzyme sequence has been attached to $5^{\prime}$ ends of aptamer sequence. Therefor its catalytic activity remained constant in all experiments. Also in their study all reagents including split DNAzyme probes, aptamer, hemin, and AB1 were added simultaneously, while our results showed that the order of addition of reagents is an important parameter.

\section{Conclusions}

Advantages of using DNAzymes and aptamers over protein enzymes and antibodies have been reported in many studies. In this study, we optimized a colorimetric simple assay using DNAzyme-aptamer conjugate to detect AFB1. Under optimized conditions, the formation of AFB1 aptamer complex prevents the hybridization of its complementary sequences. Hence, the catalytic activity of DNAzyme increases corresponding to AFB1 concentration. To improve the procedure, we will work on the limit of detection and sensitivity of this aptasensor for a more accurate and sensitive determination of AFB1.

\section{Conflicts of Interest}

The authors declare that they have no conflicts of interest.

\section{Acknowledgments}

This work was supported by a Grant (U-94080) from the Ahvaz Jundishapur University of Medical Sciences, Ahvaz, Iran.

\section{References}

[1] S. Marin, A. J. Ramos, G. Cano-Sancho, and V. Sanchis, "Mycotoxins: occurrence, toxicology, and exposure assessment," Food and Chemical Toxicology, vol. 60, pp. 218-237, 2013.

[2] M. A. Gacem and A. Ould El Hadj-Khelil, "Toxicology, biosynthesis, bio-control of aflatoxin and new methods of detection," Asian Pacific Journal of Tropical Biomedicine, vol. 6, no. 9, pp. 808-814, 2016.

[3] S. Z. Iqbal, M. R. Asi, and A. Ariño, "Aflatoxins," in Reference Module in Life Sciences, Elsevier, 2017.
[4] M. E. Smela, S. S. Currier, E. A. Bailey, and J. M. Essigmann, "The chemistry and biology of aflatoxin $\mathrm{B}_{1}$ : from mutational spectrometry to carcinogenesis," Carcinogenesis, vol. 22, no. 4, pp. 535-545, 2001.

[5] A. P. Wacoo, D. Wendiro, P. C. Vuzi, and J. F. Hawumba, "Methods for detection of aflatoxins in agricultural food crops," Journal of Applied Chemistry, vol. 2014, Article ID 706291, 15 pages, 2014.

[6] M. Darmostuk, S. Rimpelova, H. Gbelcova, and T. Ruml, "Current approaches in SELEX: an update to aptamer selection technology," Biotechnology Advances, vol. 33, no. 6, pp. 1141-1161, 2014.

[7] S. Tom, H.-E. Jin, and S.-W. Lee, "Aptamers as functional bionanomaterials for sensor applications a2 - grumezescu, alexandru mihai," in Engineering of Nanobiomaterials, chapter 6, pp. 181-226, William Andrew Publishing, 2016.

[8] C. Acquah, M. K. Danquah, J. L. S. Yon, A. Sidhu, and C. M. Ongkudon, "A review on immobilised aptamers for high throughput biomolecular detection and screening," Analytica Chimica Acta, vol. 888, pp. 10-18, 2015.

[9] Y. Seok Kim, N. H. Ahmad Raston, and M. Bock Gu, "Aptamerbased nanobiosensors," Biosensors and Bioelectronics, vol. 76, pp. 2-19, 2016.

[10] R. Nezlin, "Use of aptamers in immunoassays," Molecular Immunology, vol. 70, pp. 149-154, 2016.

[11] M. Tabarzad and M. Jafari, "Trends in the design and development of specific aptamers against peptides and proteins," Protein Journal, vol. 35, no. 2, pp. 81-99, 2016.

[12] A. Chen and S. Yang, "Replacing antibodies with aptamers in lateral flow immunoassay," Biosensors and Bioelectronics, vol. 71, pp. 230-242, 2015.

[13] J. Kosman and B. Juskowiak, "Peroxidase-mimicking DNAzymes for biosensing applications: a review," Analytica Chimica Acta, vol. 707, no. 1-2, pp. 7-17, 2011.

[14] X.-H. Zhao, H.-M. Meng, L. Gong, L. Qiu, X.-B. Zhang, and W.-H. Tan, "Recent progress of DNAzyme-nanomaterial based biosensors," Chinese Journal of Analytical Chemistry, vol. 43, no. 11, pp. 1611-1619, 2015.

[15] S. Mackay, D. Wishart, J. Z. Xing, and J. Chen, "Developing trends in aptamer-based biosensor devices and their applications," IEEE Transactions on Biomedical Circuits and Systems, vol. 8, no. 1, pp. 4-14, 2014.

[16] S. M. Bone, N. E. Lima, and A. V. Todd, "DNAzyme switches for molecular computation and signal amplification," Biosensors and Bioelectronics, vol. 70, pp. 330-337, 2015.

[17] M. McKeague and M. C. Derosa, "Challenges and opportunities for small molecule aptamer development," Journal of Nucleic Acids, vol. 2012, Article ID 748913, 20 pages, 2012.

[18] M. Citartan, S. C. B. Gopinath, J. Tominaga, S.-C. Tan, and T.$\mathrm{H}$. Tang, "Assays for aptamer-based platforms," Biosensors and Bioelectronics, vol. 34, no. 1, pp. 1-11, 2012.

[19] X. Gong, J. Li, W. Zhou, Y. Xiang, R. Yuan, and Y. Chai, “Target recycling amplification for label-free and sensitive colorimetric detection of adenosine triphosphate based on un-modified aptamers and DNAzymes," Analytica Chimica Acta, vol. 828, pp. 80-84, 2014.

[20] H. Mun, E.-J. Jo, T. Li et al., "Homogeneous assay of target molecules based on chemiluminescence resonance energy transfer (CRET) using DNAzyme-linked aptamers," Biosensors and Bioelectronics, vol. 58, pp. 308-313, 2014. 
[21] C. Yang, V. Lates, B. Prieto-Simón, J.-L. Marty, and X. Yang, "Aptamer-DNAzyme hairpins for biosensing of Ochratoxin A," Biosensors and Bioelectronics, vol. 32, no. 1, pp. 208-212, 2012.

[22] C. Yang, V. Lates, B. Prieto-Simón, J.-L. Marty, and X. Yang, "Rapid high-throughput analysis of ochratoxin A by the selfassembly of DNAzyme-aptamer conjugates in wine," Talanta, vol. 116, pp. 520-526, 2013.

[23] R. Chauhan, J. Singh, T. Sachdev, T. Basu, and B. D. Malhotra, "Recent advances in mycotoxins detection," Biosensors and Bioelectronics, vol. 81, pp. 532-545, 2016.

[24] X.-H. Yang, W.-J. Kong, M.-H. Yang, M. Zhao, and Z. Ouyang, "Application of aptamer identification technology in rapid analysis of mycotoxins," Chinese Journal of Analytical Chemistry, vol. 41, no. 2, pp. 297-306, 2013.

[25] G. Castillo, K. Spinella, A. Poturnayová, M. Šnejdárková, L. Mosiello, and T. Hianik, "Detection of aflatoxin B1 by aptamerbased biosensor using PAMAM dendrimers as immobilization platform," Food Control, vol. 52, pp. 9-18, 2015.

[26] M. Zuker, "Mfold web server for nucleic acid folding and hybridization prediction," Nucleic Acids Research, vol. 31, no. 13, pp. 3406-3415, 2003.

[27] M. Jafari, M. Rezaei, H. Kalantari, M. Tabarzad, and B. Daraei, "DNAzyme-aptamer or aptamer-DNAzyme paradigm: biochemical approach for aflatoxin analysis," Biotechnology and Applied Biochemistry, In press.

[28] X. Guo, F. Wen, N. Zheng et al., "Development of an ultrasensitive aptasensor for the detection of aflatoxin B1," Biosensors and Bioelectronics, vol. 56, pp. 340-344, 2014.

[29] W. B. Shim, M. J. Kim, H. Mun, and M. G. Kim, "An aptamerbased dipstick assay for the rapid and simple detection of aflatoxin B1," Biosensors and Bioelectronics, vol. 62, pp. 288-294, 2014.

[30] W.-B. Shim, H. Mun, H.-A. Joung, J. A. Ofori, D.-H. Chung, and M.-G. Kim, "Chemiluminescence competitive aptamer assay for the detection of aflatoxin B1 in corn samples," Food Control, vol. 36, no. 1, pp. 30-35, 2014.

[31] H. Hasegawa, N. Savory, K. Abe, and K. Ikebukuro, "Methods for improving aptamer binding affinity," Molecules, vol. 21, no. 4, article 421, 2016.

[32] Y. Seok, J.-Y. Byun, W.-B. Shim, and M.-G. Kim, "A structureswitchable aptasensor for aflatoxin B1 detection based on assembly of an aptamer/split DNAzyme," Analytica Chimica Acta, vol. 886, pp. 182-187, 2015. 

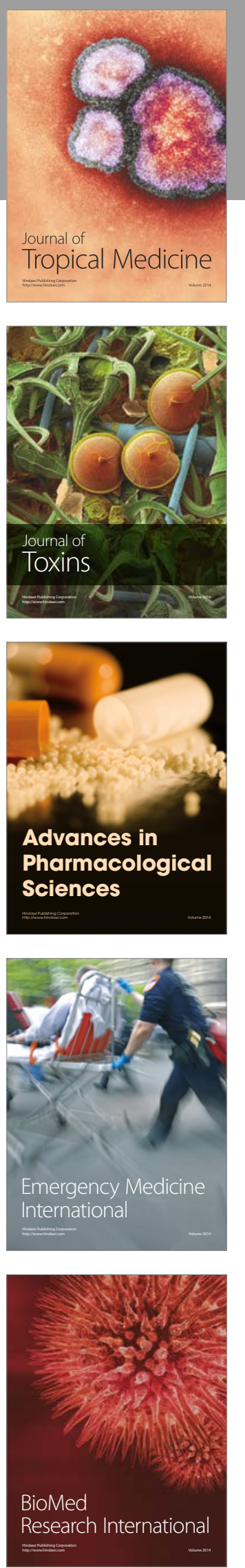
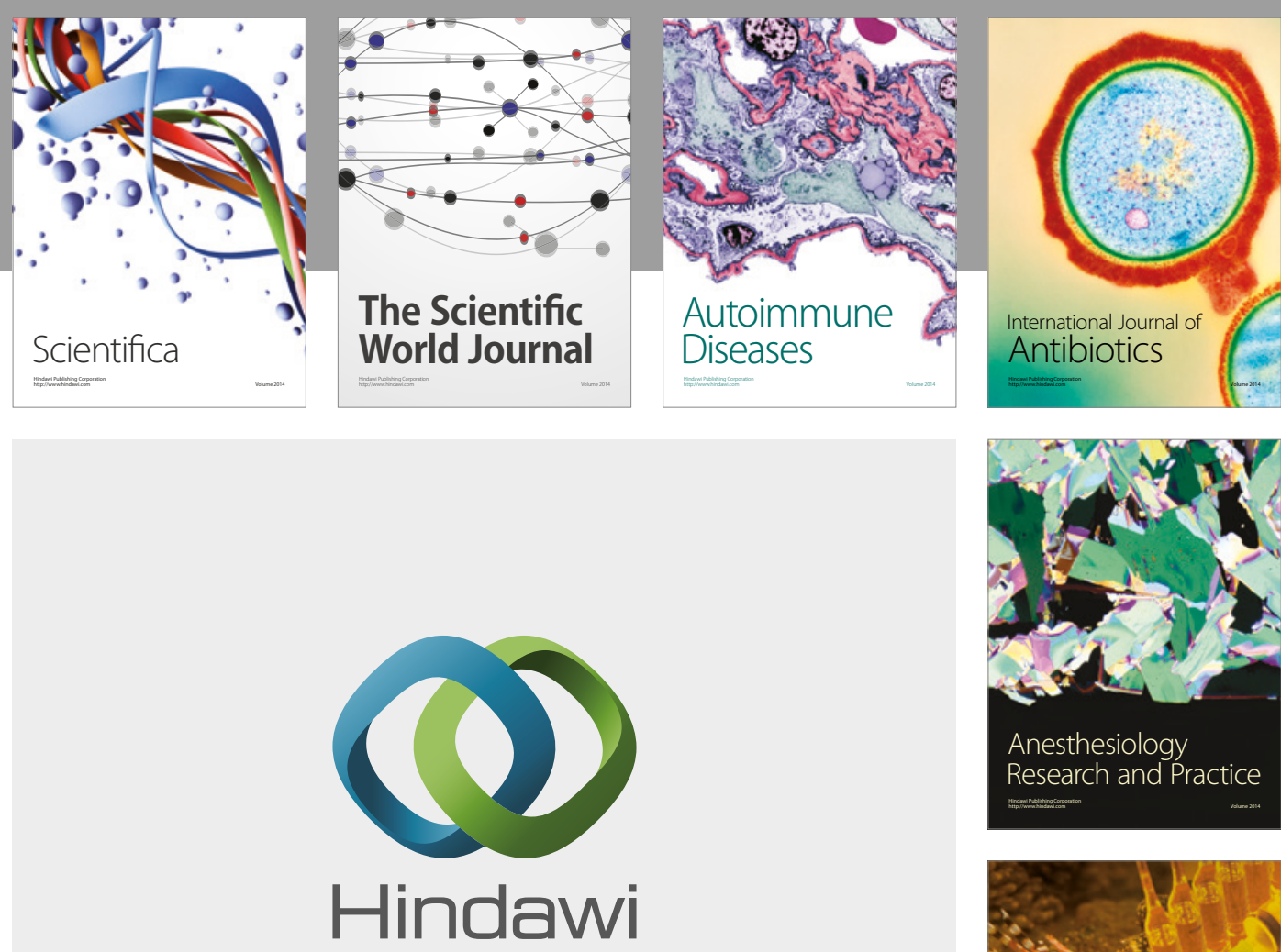

Submit your manuscripts at

https://www.hindawi.com
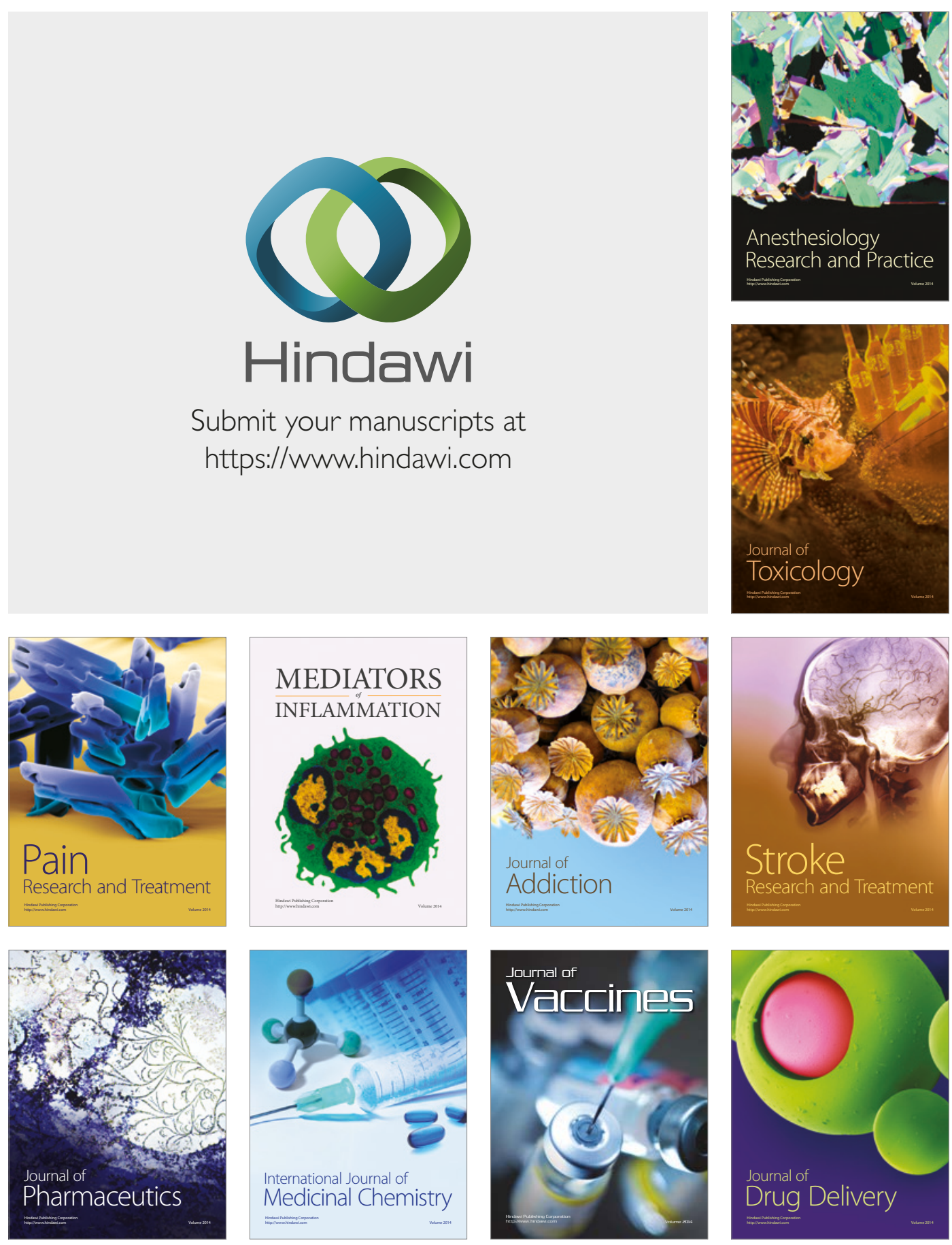